The Journal of $\mathbf{N}_{\text {onlinear }} \mathbf{S}_{\text {cience and }}$ Applications http://www.tjnsa.com

\title{
APPROXIMATION OF MIXED TYPE FUNCTIONAL EQUATIONS IN $p$-BANACH SPACES
}

\section{S. ZOLFAGHARI}

ABstraCt. In this paper, we investigate the generalized Hyers-Ulam stability of the functional equation

$$
\sum_{i=1}^{n} f\left(x_{i}-\frac{1}{n} \sum_{j=1}^{n} x_{j}\right)=\sum_{i=1}^{n} f\left(x_{i}\right)-n f\left(\frac{1}{n} \sum_{i=1}^{n} x_{i}\right) \quad(n \geq 2)
$$

in $p$-Banach spaces.

\section{INTRODUCTION AND PRELIMINARIES}

The stability problem of functional equations originated from a question of Ulam [31] in 1940, concerning the stability of group homomorphisms. Let $\left(G_{1},.\right)$ be a group and let $\left(G_{2}, *\right)$ be a metric group with the metric $d(.,$.$) . Given \epsilon>0$, does there exist a $\delta>0$, such that if a mapping $h: G_{1} \longrightarrow G_{2}$ satisfies the inequality $d(h(x . y), h(x) * h(y))<\delta$ for all $x, y \in G_{1}$, then there exists a homomorphism $H: G_{1} \longrightarrow G_{2}$ with $d(h(x), H(x))<\epsilon$ for all $x \in G_{1}$ ? In the other words, under what condition does there exists a homomorphism near an approximate homomorphism? The concept of stability for functional equation arises when we replace the functional equation by an inequality which acts as a perturbation of the equation. In 1941, D.H. Hyers [15] gave a first affirmative answer to the question of Ulam for Banach spaces. Let $f: X \longrightarrow Y$ be a mapping between Banach spaces such that

$$
\|f(x+y)-f(x)-f(y)\| \leq \delta
$$

Date: Received: 1 Feb 2010.

2000 Mathematics Subject Classification. Primary 39B82; Secondary 44B52.

Key words and phrases. Generalized Hyers-Ulam stability; Additive and Quadratic function; $p$-Banach spaces. 
for all $x, y \in X$, and for some $\delta>0$. Then there exists a unique additive mapping $A: X \longrightarrow Y$ such that

$$
\|f(x)-A(x)\| \leq \delta
$$

for all $x \in X$. Aoki [3] and Rassias [25] provided a generalization of the Hyers theorem for additive and linear functions, respectively, by allowing the Cauchy difference to be unbounded.

Theorem 1.1. (Th.M. Rassias). Let $f: X \rightarrow Y$ be a function from a normed vector space $X$ into a Banach space $Y$ subject to the inequality

$$
\|f(x+y)-f(x)-f(y)\| \leq \varepsilon\left(\|x\|^{p}+\|y\|^{p}\right)
$$

for all $x, y \in X$, where $\varepsilon$ and $p$ are constants with $\varepsilon>0$ and $p<1$. Then there exists a unique additive function $A: X \rightarrow Y$ satisfying

$$
\|f(x)-A(x)\| \leq \varepsilon\|x\|^{p} /\left(1-2^{p-1}\right)
$$

for all $x \in X$. If $p<0$ then inequality (1.1) holds for $x, y \neq 0$ and (1.2) for $x \neq 0$. Also, if for each fixed $x \in X$ the function $t \mapsto f(t x)$ is continuous in $t \in \mathbb{R}$, then $A$ is linear.

The above Theorem has provided a lot of influence during the last three decades in the development of a generalization of the Hyers-Ulam stability concept. This new concept is known as generalized Hyers-Ulam stability or Hyers-UlamRassias stability of functional equations (see [6, 16]). Furthermore, a generalization of Rassias theorem was obtained by Găvruta, who replaced $\varepsilon\left(\|x\|^{p}+\|y\|^{p}\right)$ by a general control function $\varphi(x, y)[13]$. The functional equation

$$
f(x+y)+f(x-y)=2 f(x)+2 f(y)
$$

is related to a symmetric bi-additive function [1, 22. It is natural that this equation is called a quadratic functional equation. In particular, every solution of the quadratic equation (1.3) is said to be a quadratic function. It is well known that a function $f$ between real vector spaces is quadratic if and only if there exists a unique symmetric bi-additive function $B_{1}$ such that $f(x)=B_{1}(x, x)$ for all $x$. The bi-additive function $B_{1}$ is given by

$$
B_{1}(x, y)=\frac{1}{4}(f(x+y)-f(x-y))
$$

In the paper [6], Czerwik proved the Hyers-Ulam-Rassias stability of the equation (1.3).

It was shown by Rassias [26] that the norm defined over a real vector space $X$ is induced by an inner product if and only if for a fixed integer $n \geq 2$

$$
n\left\|\frac{1}{n} \sum_{i=1}^{n} x_{i}\right\|^{2}+\sum_{i=1}^{n}\left\|x_{i}-\frac{1}{n} \sum_{j=1}^{n} x_{j}\right\|^{2}=\sum_{i=1}^{n}\left\|x_{i}\right\|^{2}
$$

for all $x_{1}, \ldots, x_{n} \in X$ (see also [2, 19]). During the last three decades a number of papers and research monographs have been published on various generalizations and applications of the generalized Hyers-Ulam stability to a number of functional equations and functions (see [5]-[14], [17, 18, 21, 22] and [26]-[29]). We also refer the readers to the books [1, 6, 16, 20, 27]. 
We consider some basic concepts concerning $p$-normed spaces.

Definition 1.2. (See [4, 30]). Let $X$ be a real linear space. A function $\|$. $\|$ : $X \rightarrow \mathbb{R}$ is a quasi-norm (valuation) if it satisfies the following conditions:

$\left(Q N_{1}\right)\|x\| \geq 0$ for all $x \in X$ and $\|x\|=0$ if and only if $x=0$;

$\left(Q N_{2}\right)\|\lambda . x\|=|\lambda| .\|x\|$ for all $\lambda \in \mathbb{R}$ and all $x \in X$;

$\left(Q N_{3}\right)$ There is a constant $M \geq 1:\|x+y\| \leq M(\|x\|+\|y\|)$ for all $x, y \in X$. Then $(X,\|\|$.$) is called a quasi-normed space. The smallest possible M$ is called the modulus of concavity of $\|$. $\|$. A quasi-Banach space is a complete quasinormed space.

A quasi-norm $\|$.$\| is called a p$-norm $(0<p \leq 1)$ if

$$
\|x+y\|^{p} \leq\|x\|^{p}+\|y\|^{p}
$$

for all $x, y \in X$. In this case, a quasi-Banach space is called a $p$-Banach space.

By the Aoki-Rolewicz Theorem [30], each quasi-norm is equivalent to some $p$-norm (see also [4]). Since it is much easier to work with $p$-norms, henceforth we restrict our attention mainly to $p$-norms.

Employing the above identity, we introduce the following functional equation deriving from additive and quadratic functions:

$$
\sum_{i=1}^{n} f\left(x_{i}-\frac{1}{n} \sum_{j=1}^{n} x_{j}\right)=\sum_{i=1}^{n} f\left(x_{i}\right)-n f\left(\frac{1}{n} \sum_{i=1}^{n} x_{i}\right)
$$

where $n \geq 2$ is a fixed integer. It is easy to see that the function $f(x)=a x^{2}+b x$ is a solution of the functional equation (1.4). A. Najati and Th. M. Rassias [23] investigated the general solution of the functional equation (1.4).

This paper is organized as follows: In Section 2, we prove the generalized Hyers-Ulam stability of the functional equation (1.4) in $p$-Banach spaces, for odd functions. The generalized Hyers-Ulam stability of the functional equation (1.4) in $p$-Banach spaces, for even functions is discussed in Section 3. Finally, in Section 4, we show that the generalized Hyers-Ulam stability of a mixed additive and quadratic functional equation (1.4) in $p$-Banach spaces.

\section{Stability of the FUnCtional equation (1.4) in $p$-Banach SPaCes: FOR ODD FUNCTIONS}

In the rest of this paper, we will assume that $X$ be a $p$-normed space and $Y$ be a $p$-Banach space. For convenience, we use the following abbreviation for a given function $f: X \rightarrow Y$,

$$
D_{f}\left(x_{1}, \ldots, x_{n}\right)=\sum_{i=1}^{n} f\left(x_{i}-\frac{1}{n} \sum_{j=1}^{n} x_{j}\right)-\sum_{i=1}^{n} f\left(x_{i}\right)+n f\left(\frac{1}{n} \sum_{i=1}^{n} x_{i}\right)
$$

for all $x_{1}, \ldots, x_{n} \in X$, where $n \geq 2$ is a fixed integer. We now investigate the generalized Hyers-Ulam stability problem for functional equation (1.4). 
Lemma 2.1. ([24]) Let $0<p \leq 1$ and let $x_{1}, x_{2}, \ldots, x_{n}$ be non-negative real numbers. Then

$$
\left(\sum_{i=1}^{n} x_{i}\right)^{p} \leq \sum_{i=1}^{n} x_{i}{ }^{p} .
$$

Theorem 2.2. Let $\ell \in\{-1,1\}$ be fixed, $X$ be a $p$-normed space, $Y$ be a $p$-Banach space and $\varphi: X^{n} \rightarrow[0, \infty)$ be a function such that

$$
\lim _{m \rightarrow \infty} 2^{m \ell} \varphi\left(\frac{x_{1}}{2^{m \ell}}, \ldots, \frac{x_{n}}{2^{m \ell}}\right)=0
$$

for all $x_{1}, \ldots, x_{n} \in X$, and

$$
\sum_{\imath=\frac{1+\ell}{2}}^{\infty} 2^{\imath p \ell} \varphi^{p}\left(\frac{u_{1}}{2^{\imath \ell}}, \ldots, \frac{u_{n}}{2^{\imath \ell}}\right)<\infty
$$

for all $u_{1} \in\{-x, x, 2 x\}$ and all $u_{2}, \ldots, u_{n} \in\{-x, 0, x\}$ (denoted $\left(\varphi\left(x_{1}, \ldots, x_{n}\right)\right)^{p}$ by $\left.\varphi^{p}\left(x_{1}, \ldots, x_{n}\right)\right)$. Suppose that an odd function $f: X \rightarrow Y$ satisfies the inequality

$$
\left\|D f\left(x_{1}, \ldots, x_{n}\right)\right\| \leq \varphi\left(x_{1}, \ldots, x_{n}\right)
$$

for all $x_{1}, \ldots, x_{n} \in X$. Furthermore, assume that $f(0)=0$ in (2.3) for the case $\ell=1$. Then the limit

$$
A(x):=\lim _{m \rightarrow \infty} 2^{m \ell} f\left(\frac{x}{2^{m \ell}}\right)
$$

exists for all $x \in X$ and $A: X \rightarrow Y$ is a unique additive function satisfying

$$
\|f(x)-A(x)\| \leq \frac{1}{2}\left(\widetilde{\psi}_{o}(x)\right)^{\frac{1}{p}}
$$

for all $x \in X$, where

$$
\begin{aligned}
\widetilde{\psi}_{o}(x):=\sum_{\imath=\frac{1+\ell}{2}}^{\infty} 2^{\imath p \ell}\left\{\varphi^{p}\left(\frac{2 x}{2^{\ell \ell}}, 0, \ldots, 0\right)+\frac{1}{2^{p}}\left[n^{p} \varphi^{p}\left(\frac{x}{2^{\imath \ell}}, \frac{x}{2^{\imath \ell}}, 0, \ldots, 0\right)\right.\right. \\
\left.\left.+\varphi^{p}\left(\frac{-x}{2^{\imath \ell}}, \frac{x}{2^{\imath \ell}}, \ldots, \frac{x}{2^{\imath \ell}}\right)+\varphi^{p}\left(\frac{x}{2^{\imath \ell}}, \frac{-x}{2^{\imath \ell}}, \ldots, \frac{-x}{2^{\imath \ell}}\right)\right]\right\}
\end{aligned}
$$

Proof. For $\ell=1$, letting $x_{1}=n x, x_{2}=-n y$ and $x_{i}=0(i=3, \ldots, n)$ in $(2.3)$ and using the oddness of $f$, we get

$$
\begin{aligned}
& \|f((n-1) x+y)-f(x+(n-1) y)-f(n x)+f(n y)+2 f(x-y)\| \\
& \quad \leq \varphi(n x,-n y, 0, \ldots, 0)
\end{aligned}
$$

for all $x, y \in X$. Letting $y=0$ in (2.7), we get

$$
\|f(n x)-f((n-1) x)-f(x)\| \leq \varphi(n x, 0, \ldots, 0)
$$

for all $x \in X$. Setting $x_{1}=n y, x_{2}=\ldots=x_{n}=n x$ in (2.3) and using the oddness of $f$, we get

$$
\begin{aligned}
\|(n-1) f(x-y) & -f((n-1)(x-y))-(n-1) f(n x)+n f((n-1) x+y) \\
& -f(n y) \| \leq \varphi(n y, n x, \ldots, n x)
\end{aligned}
$$


for all $x, y \in X$. Interchange $x$ with $y$ in (2.9) and using the oddness of $f$, we get

$$
\begin{aligned}
\| f((n-1)(x-y)) & -(n-1) f(x-y)-(n-1) f(n y)-f(n x) \\
& +n f(x+(n-1) y) \| \leq \varphi(n x, n y, \ldots, n y)
\end{aligned}
$$

for all $x, y \in X$. Using (2.7), we get from (2.9) and (2.10) that

$$
\begin{aligned}
& \|f((n-1)(x-y))+f(x-y)-f(n x)+f(n y)\| \\
& \leq \frac{1}{2}[n \varphi(n x,-n y, 0, \ldots, 0)+\varphi(n y, n x, \ldots, n x)+\varphi(n x, n y, \ldots, n y)]
\end{aligned}
$$

for all $x, y \in X$. It follows from (2.8) and (2.11) that

$$
\begin{aligned}
& \|f(n(x-y))-f(n x)+f(n y)\| \leq \varphi(n(x-y), 0, \ldots, 0) \\
& +\frac{1}{2}[n \varphi(n x,-n y, 0, \ldots, 0)+\varphi(n y, n x, \ldots, n x)+\varphi(n x, n y, \ldots, n y)]
\end{aligned}
$$

for all $x, y \in X$. Replacing $x$ by $\frac{x}{n}$ and $y$ by $\frac{-x}{n}$ in (2.12) and using the oddness of $f$, we get

$$
\begin{aligned}
\|f(2 x)-2 f(x)\| \leq \frac{1}{2}[ & n \varphi(x, x, 0, \ldots, 0)+\varphi(-x, x, \ldots, x)+\varphi(x,-x, \ldots,-x)] \\
& +\varphi(2 x, 0, \ldots, 0)
\end{aligned}
$$

for all $x \in X$. Let

$$
\begin{aligned}
\psi_{o}(x):=\frac{1}{2}[ & n \varphi(x, x, 0, \ldots, 0)+\varphi(-x, x, \ldots, x)+\varphi(x,-x, \ldots,-x)] \\
& +\varphi(2 x, 0, \ldots, 0)
\end{aligned}
$$

for all $x \in X$. Thus (2.13) means that

$$
\|f(2 x)-2 f(x)\| \leq \psi_{o}(x)
$$

for all $x \in X$. If we replace $x$ in (2.15) by $\frac{x}{2^{m+1}}$ and multiply both sides of $(2.15)$ by $2^{m}$, we see that

$$
\left\|2^{m+1} f\left(\frac{x}{2^{m+1}}\right)-2^{m} f\left(\frac{x}{2^{m}}\right)\right\| \leq 2^{m} \psi_{o}\left(\frac{x}{2^{m+1}}\right)
$$

for all $x \in X$ and all non-negative integers $m$. Hence

$$
\begin{aligned}
\left\|2^{m+1} f\left(\frac{x}{2^{m+1}}\right)-2^{k} f\left(\frac{x}{2^{k}}\right)\right\|^{p} & \leq \sum_{\imath=k}^{m}\left\|2^{\imath+1} f\left(\frac{x}{2^{\imath+1}}\right)-2^{\imath} f\left(\frac{x}{2^{\imath}}\right)\right\|^{p} \\
& \leq \sum_{\imath=k}^{m} 2^{\imath p} \psi_{o}^{p}\left(\frac{x}{2^{2+1}}\right)
\end{aligned}
$$

for all non-negative integers $m$ and $k$ with $m \geq k$ and all $x \in X$. Since $0<p \leq 1$, so by Lemma 2.1 and (2.14), we get

$$
\begin{aligned}
\psi_{o}^{p}(x) \leq \frac{1}{2^{p}} & \left.n^{p} \varphi^{p}(x, x, 0, \ldots, 0)+\varphi^{p}(-x, x, \ldots, x)+\varphi^{p}(x,-x, \ldots,-x)\right] \\
& +\varphi^{p}(2 x, 0, \ldots, 0)
\end{aligned}
$$


for all $x \in X$. Therefore it follows from (2.1), (2.2) and (2.18) that

$$
\sum_{\imath=1}^{\infty} 2^{\imath p} \psi_{o}^{p}\left(\frac{x}{2^{\imath}}\right)<\infty, \quad \lim _{m \rightarrow \infty} 2^{m} \psi_{o}\left(\frac{x}{2^{m}}\right)=0
$$

for all $x \in X$. It follows from (2.17) and (2.19) that the sequence $\left\{2^{m} f\left(\frac{x}{2^{m}}\right)\right\}$ is a Cauchy sequence for all $x \in X$. Since $Y$ is complete, the sequence $\left\{2^{m} f\left(\frac{x}{2^{m}}\right)\right\}$ converges for all $x \in X$. Therefore, one can define a function $A: X \rightarrow Y$ by

$$
A(x):=\lim _{m \rightarrow \infty} 2^{m} f\left(\frac{x}{2^{m}}\right)
$$

for all $x \in X$. Letting $k=0$ and passing the limit $m \rightarrow \infty$ in (2.17), we get

$$
\|f(x)-A(x)\|^{p} \leq \sum_{\imath=0}^{\infty} 2^{\imath p} \psi_{o}^{p}\left(\frac{x}{2^{2+1}}\right)=\frac{1}{2^{p}} \sum_{\imath=1}^{\infty} 2^{\imath p} \psi_{o}^{p}\left(\frac{x}{2^{\imath}}\right)
$$

for all $x \in X$. Therefore (2.5) follows from (2.18) and (2.21). Now we show that $A$ is additive. It follows from (2.1), (2.3) and (2.20) that

$$
\left\|D A\left(x_{1}, \ldots, x_{n}\right)\right\|=\lim _{m \rightarrow \infty} 2^{m}\left\|D f\left(\frac{x_{1}}{2^{m}}, \ldots, \frac{x_{n}}{2^{m}}\right)\right\| \leq \lim _{m \rightarrow \infty} 2^{m} \varphi\left(\frac{x_{1}}{2^{m}}, \ldots, \frac{x_{n}}{2^{m}}\right)=0
$$

for all $x_{1}, \ldots, x_{n} \in X$. Hence the function $A$ satisfies (1.4). Since $f$ is an odd function, then (2.20) implies that the function $A: X \rightarrow Y$ is odd. Therefore by Lemma 2.1 of [23], we see that the function $A: X \rightarrow Y$ is additive.

To prove the uniqueness property of $A$, let $A^{\prime}: X \rightarrow Y$ be another additive function satisfying (2.5). Since

$$
\lim _{m \rightarrow \infty} 2^{m p} \sum_{\imath=1}^{\infty} 2^{\imath p} \varphi^{p}\left(\frac{u_{1}}{2^{m+\imath}}, \ldots, \frac{u_{n}}{2^{m+\imath}}\right)=\lim _{m \rightarrow \infty} \sum_{\imath=m+1}^{\infty} 2^{\imath p} \varphi^{p}\left(\frac{u_{1}}{2^{\imath}}, \ldots, \frac{u_{n}}{2^{\imath}}\right)=0
$$

for all $u_{1} \in\{-x, x, 2 x\}$ and all $u_{2}, \ldots, u_{n} \in\{-x, 0, x\}$. Hence

$$
\lim _{m \rightarrow \infty} 2^{m p} \widetilde{\psi}_{o}\left(\frac{x}{2^{m}}\right)=0
$$

for all $x \in X$. It follows from (2.5) and (2.22) that

$$
\left\|A(x)-A^{\prime}(x)\right\|^{p}=\lim _{m \rightarrow \infty} 2^{m p}\left\|f\left(\frac{x}{2^{m}}\right)-A^{\prime}\left(\frac{x}{2^{m}}\right)\right\|^{p} \leq \frac{1}{2^{p}} \lim _{m \rightarrow \infty} 2^{m p} \widetilde{\psi}_{o}\left(\frac{x}{2^{m}}\right)=0
$$

for all $x \in X$. So we can conclude that $A(x)=A^{\prime}(x)$ for all $x \in X$. This proves the uniqueness of $A$.

For $\ell=-1$, we can prove the theorem by a similar technique.

Corollary 2.3. Let $\varepsilon, \lambda_{i}(1 \leq i \leq n)$ be non-negative real numbers such that $\lambda_{i}<1$ or $\lambda_{i}>1(1 \leq i \leq n)$. Suppose that a function $f: X \rightarrow Y$ with $f(0)=0$ satisfies

$$
\left\|D f\left(x_{1}, \ldots, x_{n}\right)\right\| \leq \varepsilon \sum_{i=1}^{n}\left\|x_{i}\right\|^{\lambda_{i}}
$$

for all $x_{1}, \ldots, x_{n} \in X$. Then there exists a unique additive function $A: X \rightarrow Y$ such that

$$
\|f(x)-A(x)\| \leq \frac{\varepsilon}{2}\left[\alpha_{1}^{p}\|x\|^{\lambda_{1} p}+\alpha_{2}^{p}\|x\|^{\lambda_{2} p}+\alpha_{3}^{p}\|x\|^{\lambda_{3} p}+\ldots+\alpha_{n}^{p}\|x\|^{\lambda_{n} p}\right]^{\frac{1}{p}}
$$


for all $x \in X$, where

$\alpha_{1}=\left[\frac{2^{p\left(1+\lambda_{1}\right)}+n^{p}+2}{\left|2^{p}-2^{\lambda_{1} p}\right|}\right]^{\frac{1}{p}}, \quad \alpha_{2}=\left[\frac{n^{p}+2}{\left|2^{p}-2^{\lambda_{2} p}\right|}\right]^{\frac{1}{p}}, \quad \alpha_{i}=\left[\frac{2^{p+1}}{\left|2^{p}-2^{\lambda_{i} p}\right|}\right]^{\frac{1}{p}} \quad(3 \leq i \leq n)$.

Proof. In Theorem 2.2, put $\varphi\left(x_{1}, \ldots, x_{n}\right):=\varepsilon \sum_{i=1}^{n}\left\|x_{i}\right\|^{\lambda_{i}}$ for all $x_{1}, \ldots, x_{n} \in$ $X$.

\section{Stability of the FUnCtional Equation (1.4) in $p$-Banach Spaces: FOR EVEN FUNCTIONS}

In this section, we prove the generalized Hyers-Ulam-Rassias stability of the functional equation (1.4) in $p$-Banach spaces for quadratic functions.

Theorem 3.1. Let $\ell \in\{-1,1\}$ be fixed, $X$ be a $p$-normed space, $Y$ be a $p$-Banach space and $\varphi: X^{n} \rightarrow[0, \infty)$ be a function such that

$$
\lim _{m \rightarrow \infty} 2^{2 m \ell} \varphi\left(\frac{x_{1}}{2^{m \ell}}, \ldots, \frac{x_{n}}{2^{m \ell}}\right)=0
$$

for all $x_{1}, \ldots, x_{n} \in X$, and

$$
\sum_{\imath=\frac{1+\ell}{2}}^{\infty} 2^{2 \imath p \ell} \varphi^{p}\left(\frac{u_{1}}{2^{\imath \ell}}, \ldots, \frac{u_{n}}{2^{\imath \ell}}\right)<\infty
$$

for all $u_{1} \in\{0, x, n x\}, u_{2} \in\{0,(n-1) x, n x\}$ and all $u_{3}, \ldots, u_{n} \in\{0, n x\}$ (denoted $\left(\varphi\left(x_{1}, \ldots, x_{n}\right)\right)^{p}$ by $\left.\varphi^{p}\left(x_{1}, \ldots, x_{n}\right)\right)$. Suppose that an even function $f: X \rightarrow Y$ satisfies the inequality

$$
\left\|D f\left(x_{1}, \ldots, x_{n}\right)\right\| \leq \varphi\left(x_{1}, \ldots, x_{n}\right)
$$

for all $x_{1}, \ldots, x_{n} \in X$. Furthermore, assume that $f(0)=0$ in (3.3) for the case $\ell=1$. Then the limit

$$
Q(x):=\lim _{m \rightarrow \infty} 2^{2 m \ell} f\left(\frac{x}{2^{m \ell}}\right)
$$

exists for all $x \in X$ and $Q: X \rightarrow Y$ is a unique quadratic function satisfying

$$
\|f(x)-Q(x)\| \leq \frac{1}{2^{2}}\left(\widetilde{\psi}_{e}(x)\right)^{\frac{1}{p}}
$$

for all $x \in X$, where

$$
\begin{aligned}
\widetilde{\psi}_{e}(x):=\sum_{\imath=\frac{1+\ell}{2}}^{\infty} 2^{2 \imath p \ell} & \left\{\frac { 1 } { ( 2 n - 2 ) ^ { p } } \left[\varphi^{p}\left(\frac{n x}{2^{\imath \ell}}, \frac{n x}{2^{\imath \ell}}, 0, \ldots, 0\right)+(2 n+4)^{p} \varphi^{p}\left(\frac{n x}{2^{\imath \ell}}, 0, \ldots, 0\right)\right.\right. \\
& \left.\left.+2^{p} \varphi^{p}\left(0, \frac{n x}{2^{\imath \ell}}, \ldots, \frac{n x}{2^{\imath \ell}}\right)+2^{p} \varphi^{p}\left(\frac{x}{2^{\imath \ell}}, \frac{(n-1) x}{2^{\imath \ell}}, 0, \ldots, 0\right)\right]\right\} .
\end{aligned}
$$

Proof. For $\ell=1$, letting $x_{1}=n x, x_{2}=-n y$ and $x_{i}=0(i=3, \ldots, n)$ in $(3.3)$ and using the evenness of $f$, we get

$$
\begin{aligned}
& \|f((n-1) x+y)+f(x+(n-1) y)-f(n x)-f(n y)+(2 n-2) f(x-y)\| \\
& \quad \leq \varphi(n x,-n y, 0, \ldots, 0)
\end{aligned}
$$

for all $x, y \in X$. Putting $y=0$ in (3.7) and using the evenness of $f$, we get

$$
\|f(n x)-f((n-1) x)-(2 n-1) f(x)\| \leq \varphi(n x, 0, \ldots, 0)
$$


for all $x \in X$. Letting $y=(1-n) x$ in (3.7) and replacing $x$ by $\frac{x}{n}$ in the obtained inequality, we get

$$
\|f((n-1) x)-f((n-2) x)-(2 n-3) f(x)\| \leq \varphi(x,(n-1) x, 0, \ldots, 0)
$$

for all $x, y \in X$. Letting $x_{1}=n x, x_{2}=\ldots=x_{n}=n y$ in (3.3) and using the evenness of $f$, we get

$$
\begin{aligned}
\| f((n-1)(x-y)) & +(n-1) f(x-y)-(n-1) f(n y)-f(n x) \\
& +n f(x+(n-1) y) \| \leq \varphi(n x, n y, \ldots, n y)
\end{aligned}
$$

for all $x, y \in X$. Since $f$ is even, it follows from (3.10) that

$$
\begin{aligned}
\| f((n-1)(x-y)) & +(n-1) f(x-y)-(n-1) f(n x)-f(n y) \\
& +n f((n-1) x+y) \| \leq \varphi(n y, n x, \ldots, n x)
\end{aligned}
$$

for all $x, y \in X$. Applying (3.7), (3.10) and (3.11), we get

$$
\begin{aligned}
\| f((n-1)(x-y)) & -(n-1)^{2} f(x-y) \| \leq \frac{1}{2}[n \varphi(n x,-n y, 0, \ldots, 0) \\
& +\varphi(n x, n y, \ldots, n y)+\varphi(n y, n x, \ldots, n x)]
\end{aligned}
$$

for all $x, y \in X$. Therefore

$$
\left\|f((n-1) x)-(n-1)^{2} f(x)\right\| \leq \frac{1}{2}[(n+1) \varphi(n x, 0, \ldots, 0)+\varphi(0, n x, \ldots, n x)]
$$

for all $x \in X$. So we get from (3.8) and (3.9)

$$
\left\|f(n x)-n^{2} f(x)\right\| \leq \frac{1}{2}[(n+3) \varphi(n x, 0, \ldots, 0)+\varphi(0, n x, \ldots, n x)]
$$

and

$$
\begin{gathered}
\left\|f((n-2) x)-(n-2)^{2} f(x)\right\| \leq \frac{1}{2}[(n+1) \varphi(n x, 0, \ldots, 0)+\varphi(0, n x, \ldots, n x)] \\
+\varphi(x,(n-1) x, 0, \ldots, 0)
\end{gathered}
$$

for all $x \in X$. Letting $y=-x$ in (3.7) and using (3.13) and (3.14), we get

$$
\begin{aligned}
\|f(2 x)-4 f(x)\| \leq \frac{1}{(2 n-2)}[\varphi(n x, n x, 0, \ldots, 0)+(2 n+4) \varphi(n x, 0, \ldots, 0) \\
+2 \varphi(0, n x, \ldots, n x)+2 \varphi(x,(n-1) x, 0, \ldots, 0)]
\end{aligned}
$$

for all $x \in X$. Let

$$
\begin{gathered}
\psi_{e}(x):=\frac{1}{(2 n-2)}[\varphi(n x, n x, 0, \ldots, 0)+(2 n+4) \varphi(n x, 0, \ldots, 0)+2 \varphi(0, n x, \ldots, n x) \\
+2 \varphi(x,(n-1) x, 0, \ldots, 0)]
\end{gathered}
$$

for all $x \in X$. Thus (3.15) means that

$$
\|f(2 x)-4 f(x)\| \leq \psi_{e}(x)
$$

for all $x \in X$. If we replace $x$ in (3.17) by $\frac{x}{2^{m+1}}$ and multiply both sides of (3.17) by $2^{2 m}$, then we have

$$
\left\|2^{2(m+1)} f\left(\frac{x}{2^{m+1}}\right)-2^{2 m} f\left(\frac{x}{2^{m}}\right)\right\| \leq 2^{2 m} \psi_{e}\left(\frac{x}{2^{m+1}}\right)
$$


for all $x \in X$ and all non-negative integers $m$. Hence

$$
\begin{aligned}
\left\|2^{2(m+1)} f\left(\frac{x}{2^{m+1}}\right)-2^{2 k} f\left(\frac{x}{2^{k}}\right)\right\|^{p} & \leq \sum_{\imath=k}^{m}\left\|2^{2(\imath+1)} f\left(\frac{x}{2^{\imath+1}}\right)-2^{2 \imath} f\left(\frac{x}{2^{\imath}}\right)\right\|^{p} \\
& \leq \sum_{\imath=k}^{m} 2^{2 \imath p} \psi_{e}^{p}\left(\frac{x}{2^{\imath+1}}\right)
\end{aligned}
$$

for all non-negative integers $m$ and $k$ with $m \geq k$ and all $x \in X$. Since $0<p \leq 1$, so by Lemma 2.1 and (3.16), we get

$$
\begin{aligned}
\psi_{e}^{p}(x) \leq \frac{1}{(2 n-2)^{p}}\left[\varphi^{p}(n x, n x, 0, \ldots, 0)+(2 n+4)^{p} \varphi^{p}(n x, 0, \ldots, 0)\right. \\
\left.+2^{p} \varphi^{p}(0, n x, \ldots, n x)+2^{p} \varphi^{p}(x,(n-1) x, 0, \ldots, 0)\right]
\end{aligned}
$$

for all $x \in X$. Therefore by (3.1), (3.2) and (3.20) we have

$$
\sum_{\imath=1}^{\infty} 2^{2 \imath p} \psi_{e}^{p}\left(\frac{x}{2^{\imath}}\right)<\infty, \quad \quad \lim _{m \rightarrow \infty} 2^{2 m} \psi_{e}\left(\frac{x}{2^{m}}\right)=0
$$

for all $x \in X$. Therefore we conclude from (3.19) and (3.21) that the sequence $\left\{2^{2 m} f\left(\frac{x}{2^{m}}\right)\right\}$ is a Cauchy sequence for all $x \in X$. Since $Y$ is complete, the sequence $\left\{2^{2 m} f\left(\frac{x}{2^{m}}\right)\right\}$ converges for all $x \in X$. So one can define the function $Q: X \rightarrow Y$ by (3.4) for all $x \in X$. Letting $k=0$ and passing the limit $m \rightarrow \infty$ in (3.19), we get

$$
\|f(x)-Q(x)\|^{p} \leq \sum_{\imath=0}^{\infty} 2^{2 \imath p} \psi_{e}^{p}\left(\frac{x}{2^{2+1}}\right)=\frac{1}{2^{2 p}} \sum_{\imath=1}^{\infty} 2^{2 \imath p} \psi_{e}^{p}\left(\frac{x}{2^{\imath}}\right)
$$

for all $x \in X$. Therefore (3.5) follows from (3.20) and (3.22). Now we show that $Q$ is quadratic. It follows from (3.1), (3.3) and (3.4) that

$$
\left\|D Q\left(x_{1}, \ldots, x_{n}\right)\right\|=\lim _{m \rightarrow \infty} 2^{2 m}\left\|D f\left(\frac{x_{1}}{2^{m}}, \ldots, \frac{x_{n}}{2^{m}}\right)\right\| \leq \lim _{m \rightarrow \infty} 2^{2 m} \varphi\left(\frac{x_{1}}{2^{m}}, \ldots, \frac{x_{n}}{2^{m}}\right)=0
$$

for all $x_{1}, \ldots, x_{n} \in X$. Therefore the function $Q$ satisfies (1.4). Since $f$ is an even function, then (3.4) implies that the function $Q: X \rightarrow Y$ is even. Therefore by Lemma 2.2 of [23], we get that the function $Q: X \rightarrow Y$ is quadratic.

To prove the uniqueness property of $Q$, let $Q^{\prime}: X \rightarrow Y$ be another quadratic function satisfying (3.5). Since

$$
\lim _{m \rightarrow \infty} 2^{2 m p} \sum_{\imath=1}^{\infty} 2^{2 \imath p} \varphi^{p}\left(\frac{u_{1}}{2^{m+\imath}}, \ldots, \frac{u_{n}}{2^{m+\imath}}\right)=\lim _{m \rightarrow \infty} \sum_{\imath=m+1}^{\infty} 2^{2 \imath p} \varphi^{p}\left(\frac{u_{1}}{2^{\imath}}, \ldots, \frac{u_{n}}{2^{\imath}}\right)=0
$$

for all $u_{1} \in\{0, x, n x\}, u_{2} \in\{0,(n-1) x, n x\}$ and all $u_{3}, \ldots, u_{n} \in\{0, n x\}$, then

$$
\lim _{m \rightarrow \infty} 2^{2 m p} \widetilde{\psi}_{e}\left(\frac{x}{2^{m}}\right)=0
$$

for all $x \in X$. Therefore it from (3.5) and the last equation that

$$
\left\|Q(x)-Q^{\prime}(x)\right\|^{p}=\lim _{m \rightarrow \infty} 2^{2 m p}\left\|f\left(\frac{x}{2^{m}}\right)-Q^{\prime}\left(\frac{x}{2^{m}}\right)\right\|^{p} \leq \frac{1}{2^{2 p}} \lim _{m \rightarrow \infty} 2^{2 m p} \widetilde{\psi_{e}}\left(\frac{x}{2^{m}}\right)=0
$$

for all $x \in X$. So we can conclude that $Q(x)=Q^{\prime}(x)$ for all $x \in X$. This proves the uniqueness of $Q$. 
For $\ell=-1$, we can prove the theorem by a similar technique.

Corollary 3.2. Let $\varepsilon, \lambda_{i}(1 \leq i \leq n)$ be non-negative real numbers such that $\lambda_{i}<2$ or $\lambda_{i}>2(1 \leq i \leq n)$. Suppose that a function $f: X \rightarrow Y$ with $f(0)=0$ satisfies

$$
\left\|D f\left(x_{1}, \ldots, x_{n}\right)\right\| \leq \varepsilon \sum_{i=1}^{n}\left\|x_{i}\right\|^{\lambda_{i}}
$$

for all $x_{1}, \ldots, x_{n} \in X$. Then there exists a unique quadratic function $Q: X \rightarrow Y$ such that

$$
\|f(x)-Q(x)\| \leq \frac{\varepsilon}{(2 n-2)}\left[\beta_{1}^{p}\|x\|^{\lambda_{1} p}+\ldots+\beta_{n}^{p}\|x\|^{\lambda_{n} p}\right]^{\frac{1}{p}}
$$

for all $x \in X$, where

$$
\begin{aligned}
& \beta_{1}=\left[\frac{n^{p \lambda_{1}}+(2 n+4)^{p} n^{p \lambda_{1}}+2^{p}}{\left|2^{2 p}-2^{p \lambda_{1}}\right|}\right]^{\frac{1}{p}}, \quad \beta_{2}=\left[\frac{n^{p \lambda_{2}}+2^{p} n^{p \lambda_{2}}+2^{p}(n-1)^{p \lambda_{2}}}{\left|2^{2 p}-2^{p \lambda_{1}}\right|}\right]^{\frac{1}{p}}, \\
& \beta_{i}=\left[\frac{2^{p} n^{p \lambda_{i}}}{\left|2^{2 p}-2^{p \lambda_{1}}\right|}\right]^{\frac{1}{p}} \quad(3 \leq i \leq n) .
\end{aligned}
$$

\section{Stability of a MiXed QUADRATIC AND ADDitive FUnCTIONAL EQUATION (1.4) IN $p$-BANACH SPACE}

Now, we are ready to prove the main theorem concerning the stability problem for functional equation (1.4) in $p$-Banach spaces.

Theorem 4.1. Let $\varphi: X^{n} \rightarrow[0, \infty)$ be a function which satisfies (2.1) for all $x_{1}, \ldots, x_{n} \in X$ and (2.2) for all $u_{1} \in\{-x, x, 2 x\}, u_{2}, \ldots, u_{n} \in\{-x, 0, x\}$ and satisfies (3.1) for all $x_{1}, \ldots, x_{n} \in X$ and (3.2) for all $u_{1} \in\{0, x, n x\}, u_{2} \in\{0,(n-$ 1) $x, n x\}$ and all $u_{3}, \ldots, u_{n} \in\{0, n x\}$. Suppose that a function $f: X \rightarrow Y$ with $f(0)=0$ satisfies the inequality (2.3) for all $x_{1}, \ldots, x_{n} \in X$. Then there exist a unique quadratic function $Q: X \rightarrow Y$ and a unique additive function $A: X \rightarrow Y$ such that

$$
\|f(x)-A(x)-Q(x)\| \leq \frac{1}{2^{3}}\left\{\left[\widetilde{\psi}_{e}(x)+\widetilde{\psi}_{e}(-x)\right]^{\frac{1}{p}}\right\}+\frac{1}{2^{2}}\left\{\left[\widetilde{\psi}_{o}(x)+\widetilde{\psi}_{o}(-x)\right]^{\frac{1}{p}}\right\}
$$

for all $x \in X$, where $\widetilde{\psi}_{e}(x)$ and $\widetilde{\psi}_{o}(x)$ are defined as in equations (2.6) and (3.6).

Proof. Assume that $\varphi: X^{n} \rightarrow[0, \infty)$ satisfies (3.1) for all $x_{1}, \ldots, x_{n} \in X$ and (3.2) for all $u_{1} \in\{0, x, n x\}, u_{2} \in\{0,(n-1) x, n x\}$ and all $u_{3}, \ldots, u_{n} \in\{0, n x\}$. Let $f_{e}(x)=\frac{1}{2}(f(x)+f(-x))$ for all $x \in X$, then $f_{e}(0)=0, f_{e}(-x)=f_{e}(x)$, and

$$
\left\|D f_{e}\left(x_{1}, \ldots, x_{n}\right)\right\| \leq \widetilde{\varphi}\left(x_{1}, \ldots, x_{n}\right)
$$

for all $x_{1}, \ldots, x_{n} \in X$, where $\widetilde{\varphi}\left(x_{1}, \ldots, x_{n}\right):=\frac{1}{2}\left(\varphi\left(x_{1}, \ldots, x_{n}\right)+\varphi\left(-x_{1}, \ldots,-x_{n}\right)\right)$. So

$$
\lim _{m \rightarrow \infty} 2^{2 m \ell} \widetilde{\varphi}\left(\frac{x_{1}}{2^{m \ell}}, \ldots, \frac{x_{n}}{2^{m \ell}}\right)=0
$$

for all $x_{1}, \ldots, x_{n} \in X$. Since

$$
\widetilde{\varphi}^{p}\left(x_{1}, \ldots, x_{n}\right) \leq \frac{1}{2^{p}}\left(\varphi^{p}\left(x_{1}, \ldots, x_{n}\right)+\varphi^{p}\left(-x_{1}, \ldots,-x_{n}\right)\right)
$$


for all $x_{1}, \ldots, x_{n} \in X$, then

$$
\sum_{\imath=\frac{1+\ell}{2}}^{\infty} 2^{2 \imath p \ell} \widetilde{\varphi}^{p}\left(\frac{u_{1}}{2^{\imath \ell}}, \ldots, \frac{u_{n}}{2^{\imath \ell}}\right)<\infty
$$

for all $u_{1} \in\{0, x, n x\}, u_{2} \in\{0,(n-1) x, n x\}$ and all $u_{3}, \ldots, u_{n} \in\{0, n x\}$. Hence from Theorem 3.1, there exists a unique quadratic function $Q: X \rightarrow Y$ such that

$$
\left\|f_{e}(x)-Q(x)\right\| \leq \frac{1}{2^{2}}\left(\tilde{\bar{\psi}}_{e}(x)\right)^{\frac{1}{p}}
$$

for all $x \in X$, where

$$
\begin{aligned}
\tilde{\widetilde{\psi}}_{e}(x):=\sum_{\imath=\frac{1+\ell}{2}}^{\infty} 2^{2 \imath p \ell}\left\{\frac { 1 } { ( 2 n - 2 ) ^ { p } } \left[\widetilde{\varphi}^{p}\left(\frac{n x}{2^{\imath \ell}}, \frac{n x}{2^{\imath \ell}}, 0, \ldots, 0\right)+(2 n+4)^{p} \widetilde{\varphi}^{p}\left(\frac{n x}{2^{\imath \ell}}, 0, \ldots, 0\right)\right.\right. \\
\left.\left.+2^{p} \widetilde{\varphi}^{p}\left(0, \frac{n x}{2^{\imath \ell}}, \ldots, \frac{n x}{2^{\imath \ell}}\right)+2^{p} \widetilde{\varphi}^{p}\left(\frac{x}{2^{\imath \ell}}, \frac{(n-1) x}{2^{\imath \ell}}, 0, \ldots, 0\right)\right]\right\}
\end{aligned}
$$

for all $x \in X$. It is clear that

$$
\tilde{\bar{\psi}}_{e}(x) \leq \frac{1}{2^{p}}\left[\widetilde{\psi}_{e}(x)+\widetilde{\psi}_{e}(-x)\right]
$$

for all $x \in X$. Therefore it follows from (4.2) that

$$
\left\|f_{e}(x)-Q(x)\right\| \leq \frac{1}{2^{3}}\left[\widetilde{\psi}_{e}(x)+\widetilde{\psi}_{e}(-x)\right]^{\frac{1}{p}}
$$

for all $x \in X$.

Also, let $f_{o}(x)=\frac{1}{2}(f(x)-f(-x))$ for all $x \in X$, by using the above method and Theorem 2.2, it follows that there exist a unique additive function $A: X \rightarrow Y$ such that

$$
\left\|f_{o}(x)-A(x)\right\| \leq \frac{1}{2^{2}}\left[\widetilde{\psi}_{o}(x)+\widetilde{\psi}_{o}(-x)\right]^{\frac{1}{p}}
$$

for all $x \in X$. Hence (4.1) follows from (4.3) and (4.4). Now, if $\varphi: X^{n} \rightarrow[0, \infty)$ satisfies (2.1) for all $x_{1}, \ldots, x_{n} \in X$ and (2.2) for all $u_{1} \in\{-x, x, 2 x\}$ and all $u_{2}, \ldots u_{n} \in\{-x, 0, x\}$, we can prove the theorem by a similar technique.

Corollary 4.2. Let $\varepsilon, \lambda_{i}(1 \leq i \leq n)$ be non-negative real numbers such that $1<\lambda_{i}<2$ or $\lambda_{i}>2$ or $\lambda_{i}<1(1 \leq i \leq n)$. Suppose that a function $f: X \rightarrow Y$ satisfies the inequality $\left\|D f\left(x_{1}, \ldots, x_{n}\right)\right\| \leq \varepsilon \sum_{i=1}^{n}\left\|x_{i}\right\|^{\lambda_{i}}$ for all $x_{1}, \ldots, x_{n} \in X$. Furthermore, assume that $f(0)=0$ for the case $f$ is even. Then there exist a unique quadratic function $Q: X \rightarrow Y$ and a unique additive function $A: X \rightarrow Y$ such that

$$
\begin{aligned}
\|f(x)-Q(x)-A(x)\| & \leq \frac{\varepsilon}{(2 n-2)}\left[\beta_{1}^{p}\|x\|^{\lambda_{1} p}+\ldots+\beta_{n}^{p}\|x\|^{\lambda_{n} p}\right]^{\frac{1}{p}} \\
& +\frac{\varepsilon}{2}\left[\alpha_{1}^{p}\|x\|^{\lambda_{1} p}+\ldots+\alpha_{n}^{p}\|x\|^{\lambda_{n} p}\right]^{\frac{1}{p}}
\end{aligned}
$$

for all $x \in X$, where $\alpha_{i}$ and $\beta_{i}(1 \leq i \leq n)$ are defined as in Corollaries (2.3) and (3.2). 
Proof. put $\varphi\left(x_{1}, \ldots, x_{n}\right):=\varepsilon \sum_{i=1}^{n}\left\|x_{i}\right\|^{\lambda_{i}}$, Since

$$
\left\|D_{f_{e}}\left(x_{1}, \ldots, x_{n}\right)\right\| \leq \varphi\left(x_{1}, \ldots, x_{n}\right), \quad\left\|D_{f_{o}}\left(x_{1}, \ldots, x_{n}\right)\right\| \leq \varphi\left(x_{1}, \ldots, x_{n}\right)
$$

for all $x_{1}, \ldots, x_{n} \in X$. Thus the result follows from Corollaries (2.3) and (3.2).

\section{REFERENCES}

[1] J. Aczel and J. Dhombres, Functional Equations in Several Variables, Cambridge University Press, 1989.

[2] D. Amir, Characterizations of Inner Product Spaces, Birkhuser, Basel, 1986.

[3] T. Aoki, On the stability of the linear transformation in Banach spaces, J. Math. Soc. Japan. 2 (1950) 64-66.

[4] Y. Benyamini and J. Lindenstrauss, Geometric Nonlinear Functional Analysis, vol. 1, Colloq. Publ. vol. 48, Amer. Math. Soc., Providence, RI, 2000.

[5] P.W. Cholewa, Remarks on the stability of functional equations, Aequationes Math. 27 (1984) 76-86.

[6] P. Czerwik, Functional Equations and Inequalities in Several Variables, World Scientific Publishing Company, New Jersey, Hong Kong, Singapore, London, 2002.

[7] S. Czerwik, On the stability of the quadratic mapping in normed spaces, Abh. Math. Sem. Univ. Hamburg. 62 (1992) 59-64.

[8] M. Eshaghi Gordji and H. Khodaei, Solution and stability of generalized mixed type cubic, quadratic and additive functional equation in quasi-Banach spaces, Nonlinear Analysis.TMA. 71 (2009) 5629-5643.

[9] M. Eshaghi Gordji and H. Khodaei, On the Generalized Hyers-Ulam-Rassias Stability of Quadratic Functional Equations, Abstract and Applied Analysis Volume 2009, Article ID 923476, 11 pages.

[10] M. Eshaghi Gordji, H. Khodaei and C. Park, A fixed point approach to the Cauchy-Rassias stability of general Jensen type quadratic-quadratic mappings, (To appear).

[11] V.A. Faizev, Th.M. Rassias and P.K. Sahoo, The space of $(\psi, \gamma)$-additive mappings on semigroups, Trans. Amer. Math. Soc. 354 (11) (2002) 4455-4472.

[12] Z. Gajda, On stability of additive mappings, Int. J. Math. Math. Sci. 14 (1991) 431-434.

[13] P. Gcheckavruta, A generalization of the Hyers-Ulam-Rassias stability of approximately additive mappings, J. Math. Anal. Appl. 184 (1994) 431-436.

[14] A. Grabiec, The generalized Hyers-Ulam stability of a class of functional equations, Publ. Math. Debrecen. 48 (1996) 217-235.

[15] D.H. Hyers, On the stability of the linear functional equation, Proc. Natl. Acad. Sci. 27 (1941) 222-224.

[16] D.H. Hyers, G. Isac and Th.M. Rassias, Stability of Functional Equations in Several Variables, Birkhauser, Basel, 1998.

[17] D.H. Hyers and Th.M. Rassias, Approximate homomorphisms, Aequationes Math. 44 (1992) 125-153.

[18] G. Isac and Th.M. Rassias, Stability of $\psi$-additive mappings: Applications to nonlinear analysis, Internat. J. Math. Math. Sci. 19 (1996) 219-228.

[19] P. Jordan and J. Neumann, On inner products in linear metric spaces, Ann. Math. 36 (1935) 719-723.

[20] S.-M. Jung, Hyers-Ulam-Rassias Stability of Functional Equations in Mathematical Analysis, Hadronic Press Inc., Palm Harbor, Florida, 2001.

[21] S.-M. Jung and T.-S. Kim, A fixed point approach to stability of cubic functional equation, Bol. Soc. Mat. Mexicana 12 (2006) 51-57.

[22] Pl. Kannappan, Quadratic functional equation and inner product spaces, Results Math. 27 (1995) 368-372. 
[23] A. Najati, Th.M. Rassias, Stability of a mixed functional equation in several variables on Banach modules, Nonlinear Analysis.-TMA, in press.

[24] A. Najati, M. B. Moghimi, Stability of a functional equation deriving from quadratic and additive function in quasi-Banach spaces, J. Math. Anal. Appl. 337 (2008) 399-415.

[25] Th.M. Rassias, On the stability of the linear mapping in Banach spaces, Proc. Amer. Math. Soc. 72 (1978) 297-300.

[26] Th.M. Rassias, New characterization of inner product spaces, Bull. Sci. Math. 108 (1984) 95-99.

[27] Th.M. Rassias, Functional Equations, Inequalities and Applications, Kluwer Academic Publishers Co., Dordrecht, Boston, London, 2003.

[28] Th.M. Rassias and P. Šemrl, On the Hyers-Ulam stability of linear mappings, J. Math. Anal. Appl. 173 (1993) 325-338.

[29] Th.M. Rassias and K. Shibata, Variational problem of some quadratic functionals in complex analysis, J. Math. Anal. Appl. 228 (1998) 234-253.

[30] S. Rolewicz, Metric Linear Spaces, PWN-Polish Sci. Publ.-Reidel, Warszawa-Dordrecht, 1984.

[31] S.M. Ulam, Problems in Modern Mathematics, Chapter VI, science Editions., Wiley, New York, 1964.

Department of Mathematics, Semnan University, P. O. Box 35195-363, Semnan, IRAN.

E-mail address: somaye.zolfaghari@gmail.com 